\title{
Role of mission criticality and component reliability in defining and evaluating system effectiveness
}
A. Bender*
F. Bowden*
A. Pincombe*
P. Williams*

(Received 8 March 2006; revised 15 April 2007)

\begin{abstract}
We present a framework that not only links system survival to survival rates of system components but also to the degree to which these components contribute to the system's ability to achieve a mission. In the framework a system is made up of multiple, possibly heterogeneous, components, each having a known probability of failure. The overall measure of system effectiveness is then a function of component reliability probabilities and mission criticality. In this approach the interdependency of component reliability and mission criticality is adequately modelled, which when ignored can lead to incorrect assessments of system performance.
\end{abstract}

*Land Operations Division, Defence Science and Technology Organisation, Edinburgh, South Australia. mailto:adrian.pincombe@dsto.defence.gov.au See http://anziamj.austms.org.au/V47EMAC2005/Bender for this article, (C) Austral. Mathematical Soc. 2007. Published June 26, 2007. ISSN 1446-8735 


\section{Contents}

1 Introduction

C761

2 Framework definition

C763

2.1 Mathematical formulation . . . . . . . . . . . . C763

2.2 One component-type systems . . . . . . . . . . . . C764

2.3 System effectiveness . . . . . . . . . . . . . . C C765

2.4 Two component-type systems . . . . . . . . . . . C766

2.5 System effectiveness, reliability and option preference reversal C769

2.6 Generalisation to systems comprising components of $k$ different types . . . . . . . . . . . . . . . C C770

3 Summary and discussion

References

C774

\section{Introduction}

We define a framework that links the value of a system to the reliability of its components and the performance of the system as it degrades. It does this by taking into account the degree to which these components contribute to the system's ability to achieve a mission. In the framework the system has multiple components, each having a known reliability. The system is used to carry out a mission profile comprising a time series of tasks. System effectiveness is defined as the degree to which the system is expected to achieve the tasks in the mission profile. It is proposed that this measure of system effectiveness should be used in comparing different potential system options whenever system reliability is a critical factor.

The problem addressed in this article was motivated by a military study into Offensive Support systems. Although the work was motivated by a 
military problem the approach developed is applicable across a wide range of areas. For example, it could be equally applicable to a courier business. In this case the components would be different types of vehicles such as bicycles, cars, vans and trucks.

The work in this article arose from the analysis of a multi-criteria decision problem in which some of the criteria were not independent of each other, making standard linear methods of aggregation inapplicable $[1,2,7]$. Here we focus on the dependence between effectiveness and reliability, but we also show that some of the other criteria can be derived from an extension to our approach. A defining factor of the problems we consider is the assessment of a system against a fixed set of tasks. These tasks are given in a context where their timings are set out in a broader scenario to represent typical task ordering and task overlaps. It is the schedule of the tasks that determines the demands on the system and how well a given representation for a system is able to meet the requirements that will be placed on it.

A complication of the systems under consideration is that not all components are able to perform all the tasks. In addition, some components are better able to achieve some tasks than others. For example, in a courier system bicycles cannot be used to deliver a washing machine. A car is able to, but a van is far better suited to this task. Given that, amongst others, we like to assess system reliability, it is reasonable to look at reliability modelling for a solution to the problem. There are two classes of reliability performance models: Markov models and combinational models [4, 6]. Markov processes underpin most reliability assessment models and form the basis for our model as they have the power to model a wide variety of processes. In particular, it is Markov Reward Models that seem to best suit our problem [5, 4]. In Markov Reward Models, rewards are associated with the time spent in each state. In the limit of large time we can calculate the expected value of the rewards, based on the cumulative value of the fraction of time spent in state $i$.

Most of the reliability modelling in the literature is applied to software systems where the system either works perfectly or fails, and where the im- 
portance of a component is used to determine the level of redundancy that is required. This is a major difference in the work presented in this article. We are not so interested in conditions of full failure but the partial effectiveness of the system as components fail and the development of a MOE that reflects system performance under partial failure. We adopt the performability model [3] which combines measures of performance and reliability by introducing a capability function which links lower level behaviour to system level measures. Inspired by the performability model we develop the concept of 'mission criticality', which is a generalisation of Meyer's capability function [3] describing system effectiveness for all system states that can be reached by component failure. By convolving this mission criticality with component reliability we arrive at an expression for system performance assessment in which effectiveness and component reliability are entangled. At the end of this article we generalise this framework to include system states that can be reached by system parameter variation other than component failure.

\section{Framework definition}

In this section we define our framework. This includes outlining the mathematical formulation of the problem and shows the advantages of our approach over a standard approach.

\subsection{Mathematical formulation}

Given a system composed of $k$ different types of components. The system comprises a total of $N$ components, $N_{1}$ of which are of type $1, N_{2}$ of type 2 , etc. In order to describe the system we use the $k$-tuple $\mathbf{N}=\left(N_{1}, N_{2}, \ldots, N_{k}\right)$ with $\|\mathbf{N}\| \equiv N=\sum_{i=1}^{k} N_{i}$. Without loss of generality we construct the system such that $N_{i} \geq 1$ for all $i \in\{1, \ldots, k\}$; however, we drop this constraint 
when we generalise the developed framework in the Discussion section.

It is not necessary that all parts of the system are crucial for its performance. The system may have inbuilt redundancy, that is, the system can survive the breakdown or loss of some of its components. As before we describe the redundant aspects of the system by a $k$-tuple $\mathbf{R}=\left(R_{1}, \ldots, R_{k}\right)$ with $\|\mathbf{R}\| \equiv R=\sum_{i=1}^{k} R_{i}$ and $R_{i} \leq N_{i}$ for all $i \in\{1, \ldots, k\}$. For the purpose of motivating our approach, we assume for the moment that a component of type $i$ cannot replace a component of type $j$; however, in the framework of mission criticality, that is introduced soon, we drop this assumption.

In our analysis we want to evaluate the system's reliability or effectiveness building upon our knowledge of the of the individual system components. Let $p_{i}$ denote the probability of reliability of individual components of type $i$, and $q_{i}=1-p_{i}$ the probability of breakdown of that component type. Let $n_{i}$ denote the number of components of type $i$ that fail. Then one can start to describe system failure by the probability $Q$ of failure, when for at least one of the component types the condition $n_{i}>R_{i}$ is fulfilled. The system reliability is $P=1-Q$.

\subsection{One component-type systems}

Consider a system that comprises $N$ components of the same type $(k=1)$ with $R(<N)$ of them being redundant. In the case that the system can either perform fully (when enough, that is, more than $N-R-1$, components are functioning) or fail completely (when more than $R$ components break down), the probability of system reliability is

$$
{ }^{\mathbf{N}} P_{k=1}=\sum_{i=N-R}^{N}\left(\begin{array}{c}
N \\
i
\end{array}\right) p^{i} q^{N-i} .
$$

Here the superscript $\mathbf{N}=\left(N_{1}\right)$ is a one-tuple and is introduced in order to have a convention for generalising to many component systems. By intro- 
ducing the vector $\mathbf{w}$ whose $N$ components are

$$
w_{j} \equiv \theta_{N-R, j} \equiv \begin{cases}0, & j<N-R \\ 1, & j \geq N-R\end{cases}
$$

Equation (1) is written as

$$
{ }^{\mathbf{N}} P_{1}=\sum_{i=0}^{N}\left(\begin{array}{c}
N \\
i
\end{array}\right) p^{i} q^{N-i} w_{i}=\sum_{i=0}^{N} \tilde{P}_{i} w_{i}=\left\|\tilde{\mathbf{P}}_{\mathbf{w}}\right\|_{1} .
$$

We introduced the vector $\mathbf{w}$ as it describes the dependence of system effectiveness as a function of the number of serviceable components. In the case described so far, the system requires at least $N-R$ components to be functional; that is, if $i<N-R$ components are serviceable, then $w_{j}=0$ and the whole system fails to perform.

Because of this interpretation we term the vector $\mathbf{w}$ the 'mission criticality' vector. It reflects the importance of the functioning of a system's components in the system's ability to perform a given mission. Having made this interpretation, it is easy to generalise the performance measure introduced in Equation (3) to apply to systems in which component degradation does not result in complete system failure, or in which the system of $N$ components is unable to achieve the mission in full. We define generalised mission criticality as the components $w_{j}$ of a vector $\mathbf{w}$ obeying the constraints

$$
0 \leq w_{j} \leq 1, \quad w_{0}=0 \quad \text { and } \quad w_{j} \leq w_{j+1} .
$$

The last of these three constraints is only true for coherent systems and is relaxed when one considers systems that degrade in performance when components are added.

\subsection{System effectiveness}

Mission criticality is closely linked to system effectiveness. If we ignore, as is often done, the probability of component failure, then the effectiveness $E$ of 
a system with $N$ components of one type can, for instance, be defined as the number of completed tasks divided by the total number of tasks in a given mission, provided that the system is, and remains, in the design state. The design state is the system state in which all $N$ components are functioning. This corresponds to the value of mission criticality $w_{j}$ for $j=N$ of

$$
E=w_{N}
$$

Including the probability of component failure will reduce this effectiveness. The mission criticality vector $\mathbf{w}$ needs to be multiplied by the probability of system reliability, and one needs to take the sum over all possible states that can be reached from the design state. This is exactly what is done in Equation (3). The function ${ }^{\mathbf{N}} P$ therefore is used to define a system's effectiveness $\hat{E}$ that takes into account component reliability:

$$
\hat{E}={ }^{\mathbf{N}} P \text {. }
$$

As can be shown, if $p<1$ then $\hat{E} \leq E$ for systems fulfilling the constraints (4). Also, $\lim _{p \rightarrow 1} \hat{E}=w_{N}=E$, which shows that in the case of fully reliable components, the reliability dependent system effectiveness measure equals the effectiveness measure that ignores component reliability.

To illustrate the effect of component reliability on system effectiveness we consider one component-type systems that perform the same mission but have different numbers of components (in the example, $N$ varies from 1 to 10). The mission is described by a mission criticality of the form of a cumulative Weibull function, $w_{n}=1-\exp \left\{-(n / 3)^{2}\right\}$. Figure 1 shows how system effectiveness decreases with increasing probability of component failure, $q=1-p$, or decreasing component reliability, $p$.

\subsection{Two component-type systems}

For a system which comprises $N_{1}$ components of type 1 and $N_{2}$ components of type $2(k=2)$ with $R_{1}<N_{1}$ and $R_{2}<N_{2}$ redundant parts, and which either 


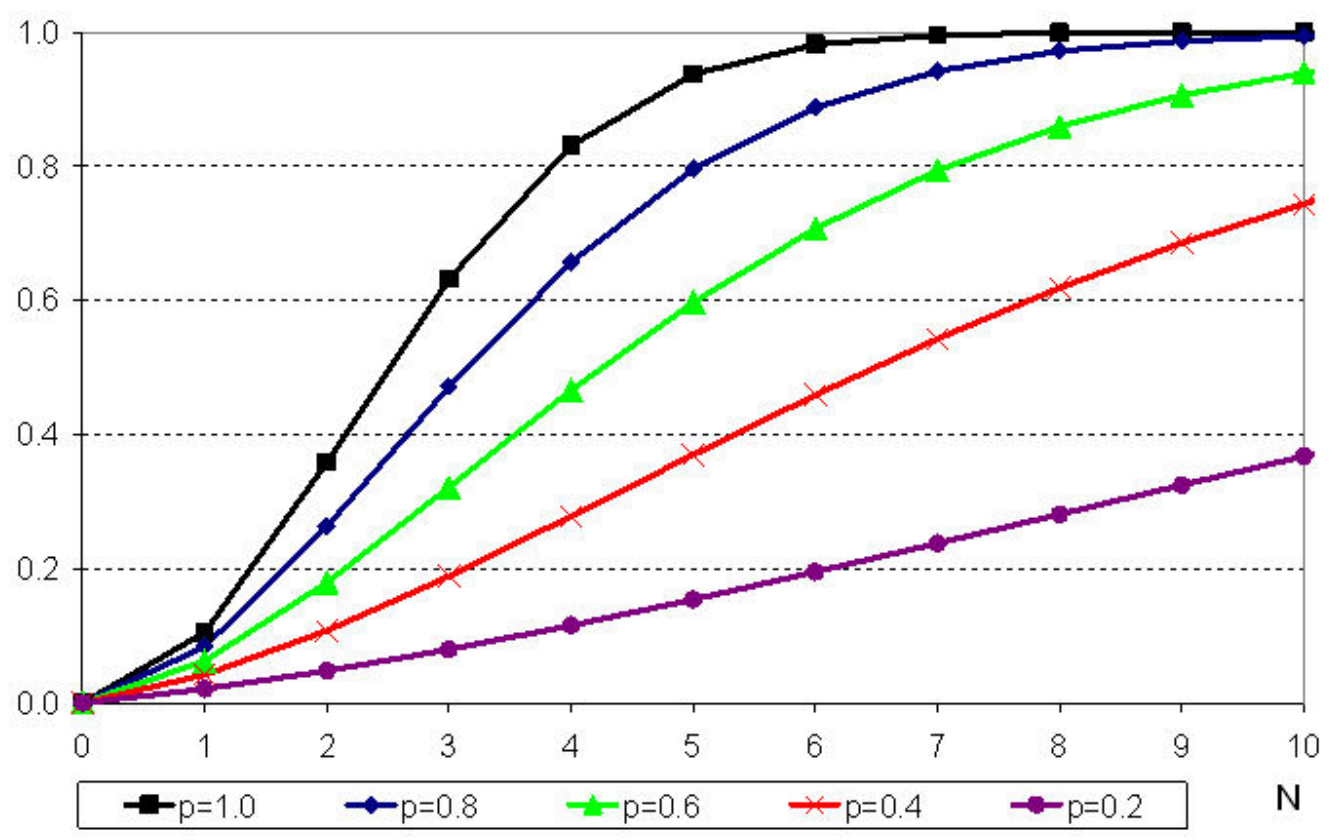

FiguRE 1: Effect of component failure on system effectiveness for a one component type system. 
is fully effective when sufficiently many components work, or fails completely when the number of failing components of a type exceeds the number of redundant parts of that type, the probability of system reliability is

$$
{ }^{\mathbf{N}} P_{k=2}=\sum_{i_{1}=N_{1}-R_{1}}^{N_{1}}\left(\begin{array}{c}
N_{1} \\
i_{1}
\end{array}\right) p_{1}^{i_{1}} q_{1}^{N_{1}-i_{1}} \sum_{i_{2}=N_{2}-R_{2}}^{N_{2}}\left(\begin{array}{c}
N_{2} \\
i_{2}
\end{array}\right) p_{2}^{i_{2}} q_{2}^{N_{2}-i_{2}} .
$$

Here the superscript $\mathbf{N}=\left(N_{1}, N_{2}\right)$ is a two-tuple. As in the case of $k=1$ we introduce the matrix $\mathbf{w}$ with components

$$
w_{j_{1}, j_{2}} \equiv \theta_{N_{1}-R_{1}, j_{1}} \theta_{N_{2}-R_{2}, j_{2}}
$$

to arrive at

$$
{ }^{\mathbf{N}} P_{2}=\sum_{i_{1}=0}^{N_{1}} \sum_{i_{2}=0}^{N_{2}}\left(\begin{array}{c}
N_{1} \\
i_{1}
\end{array}\right)\left(\begin{array}{c}
N_{2} \\
i_{2}
\end{array}\right) p_{1}^{i_{1}} q_{1}^{N_{1}-i_{1}} p_{2}^{i_{2}} q_{2}^{N_{2}-i_{2}} w_{i_{1} i_{2}}=\left\|\tilde{\mathbf{P}}^{(1)} \tilde{\mathbf{P}}^{(2)} \mathbf{W}\right\|_{1} .
$$

As in Equation (3) we use the vectors $\tilde{\mathbf{P}}$ (with the superscript denoting the component type) and matrix $\mathbf{w}$. The components of the matrix $\tilde{\mathbf{P}}^{(1)} \tilde{\mathbf{P}}^{(2)} \mathbf{W}$ are $\left(\tilde{\mathbf{P}}^{(1)} \tilde{\mathbf{P}}^{(2)} \mathbf{w}\right)_{i, j}=\tilde{P}_{i}^{(1)} \tilde{P}_{j}^{(2)} w_{i, j}$.

Once more we can generalise the concept by imposing the constraints

$$
0 \leq w_{j_{1}, j_{2}} \leq 1, \quad w_{0,0}=0, \quad w_{j_{1}, j_{2}} \leq w_{j_{1}+1, j_{2}} \quad \text { and } \quad w_{j_{1}, j_{2}} \leq w_{j_{1}, j_{2}+1}
$$

Again, this enables us to describe system performance in which component failure does not result in complete system failure, or in which the system of $N=\|\mathbf{N}\|$ components is unable to achieve the mission in full. As the generalised mission criticality matrix does not necessarily need to be the dyadic product of two mission criticality vectors, this formulation of system performance also allows us to study systems, in which components of type $i$ replace (a proportion of the functions of) type- $j$ components $(i, j \in\{1,2\}$ ). 


\subsection{System effectiveness, reliability and option preference reversal}

In this section we study an example in order to examine how the introduction of component reliability can influence the assessment of system effectiveness and change the preferred system. Firstly, we define a system-effectiveness measure $E$ that ignores component reliability and assumes that the system remains (reliably) in its design state $\left(N_{1}, N_{2}\right)$ throughout the whole mission:

$$
E=w_{N_{1}, N_{2}} .
$$

However, if component reliability is taken into account Equation (9) needs to be applied. Thus define

$$
\hat{E} \equiv{ }^{\mathbf{N}} P_{2} .
$$

If both component types are $100 \%$ reliable, then $\hat{E}=E$.

Consider two systems which both are built from components of type 1 and type 2. System 1 has design state $\left(N_{1}, N_{2}\right)=(3,2)$, and System 2 has $\left(N_{1}, N_{2}\right)=(2,3)$. Both systems are not fully effective with $(2,2)$; however, some of the tasks in the mission, namely a proportion $0<a<1$, are performed in this subsystem. None of the tasks are performed if $n_{1}<2$ or $n_{2}<2$. For System 1 (2) the reliability-independent system effectiveness increases by $0<\varepsilon_{1} \leq 1-a\left(0<\varepsilon_{2} \leq 1-a\right)$ over the $(2,2)$ subsystem. In other words, the mission criticality tensors for the two systems are

$$
\begin{aligned}
w^{\text {System 1 }} & =\left(\begin{array}{lll}
w_{00} & w_{01} & w_{02} \\
w_{10} & w_{11} & w_{12} \\
w_{20} & w_{21} & w_{22} \\
w_{30} & w_{31} & w_{32}
\end{array}\right)=\left(\begin{array}{ccc}
0 & 0 & 0 \\
0 & 0 & 0 \\
0 & 0 & a \\
0 & 0 & a+\varepsilon_{1}
\end{array}\right) \text { and } \\
w^{\text {System 2 }} & =\left(\begin{array}{cccc}
0 & 0 & 0 & 0 \\
0 & 0 & 0 & 0 \\
0 & 0 & a & a+\varepsilon_{2}
\end{array}\right) .
\end{aligned}
$$


If component reliability is not taken into account, then $E_{1}=a+\varepsilon_{1}$ and $E_{2}=a+\varepsilon_{2}$ for System 1 and System 2, respectively. If this measure is used, then System 1 is 'more effective' than System 2 if and only if $\varepsilon_{1}>\varepsilon_{2}$.

However, if component reliability is taken into account, then the appropriate measure of system effectiveness is the probability $\hat{E}={ }^{\mathbf{N}} P_{2}$ in Equation (9). In the example presented here $\hat{E}_{\text {System } 1}=a p_{1}^{2} p_{2}^{2}\left(3-2 p_{1}\right)+$ $\varepsilon_{1} p_{1}^{3} p_{2}^{2}$ and $\hat{E}_{2}=a p_{1}^{2} p_{2}^{2}\left(3-2 p_{2}\right)+\varepsilon_{2} p_{1}^{2} p_{2}^{3}$. Thus, $\hat{E}_{1}>\hat{E}_{2}$ only if $\varepsilon_{1}>$ $\frac{1}{p_{1}}\left[2 a\left(p_{1}-p_{2}\right)+\varepsilon_{2} p_{2}\right]$.

Therefore we get a reversal of the preferred system in the assessment of system effectiveness if

$$
2 a\left(1-\frac{p_{2}}{p_{1}}\right)+\varepsilon_{2} \frac{p_{2}}{p_{1}}>\varepsilon_{1}>\varepsilon_{2} .
$$

While for $a>\frac{1}{3}$ and $p_{2}>p_{1}$ these two inequalities cannot be fulfilled simultaneously, there are subspaces in the $\left(\varepsilon_{1}, \varepsilon_{2}, a, p_{2} / p_{1}\right)$ space for which both inequalities in Equation (14) are true. These subspaces are shown (as the shaded areas) in Figure 2. This example shows how the inclusion of reliability in the system effectiveness measure can impact the preferred system option. It illustrates that looking at the effectiveness without considering reliability and the reduction in system effectiveness under partial failure conditions can lead to choosing the wrong system.

\subsection{Generalisation to systems comprising components of $k$ different types}

It is straightforward to generalise the formalism developed for two componenttype systems to systems that comprise components of $k$ types. Component reliability dependent system effectiveness is defined as

$$
\hat{E} \equiv{ }^{\mathbf{N}} P_{k}=\sum_{i_{1}=0}^{N_{1}} \cdots \sum_{i_{k}=0}^{N_{k}} w_{i_{1}, \ldots, i_{k}} \prod_{j=1}^{k}\left(\begin{array}{c}
N_{j} \\
i_{j}
\end{array}\right) p_{j}^{i_{j}} q_{j}^{N_{j}-i_{j}}
$$



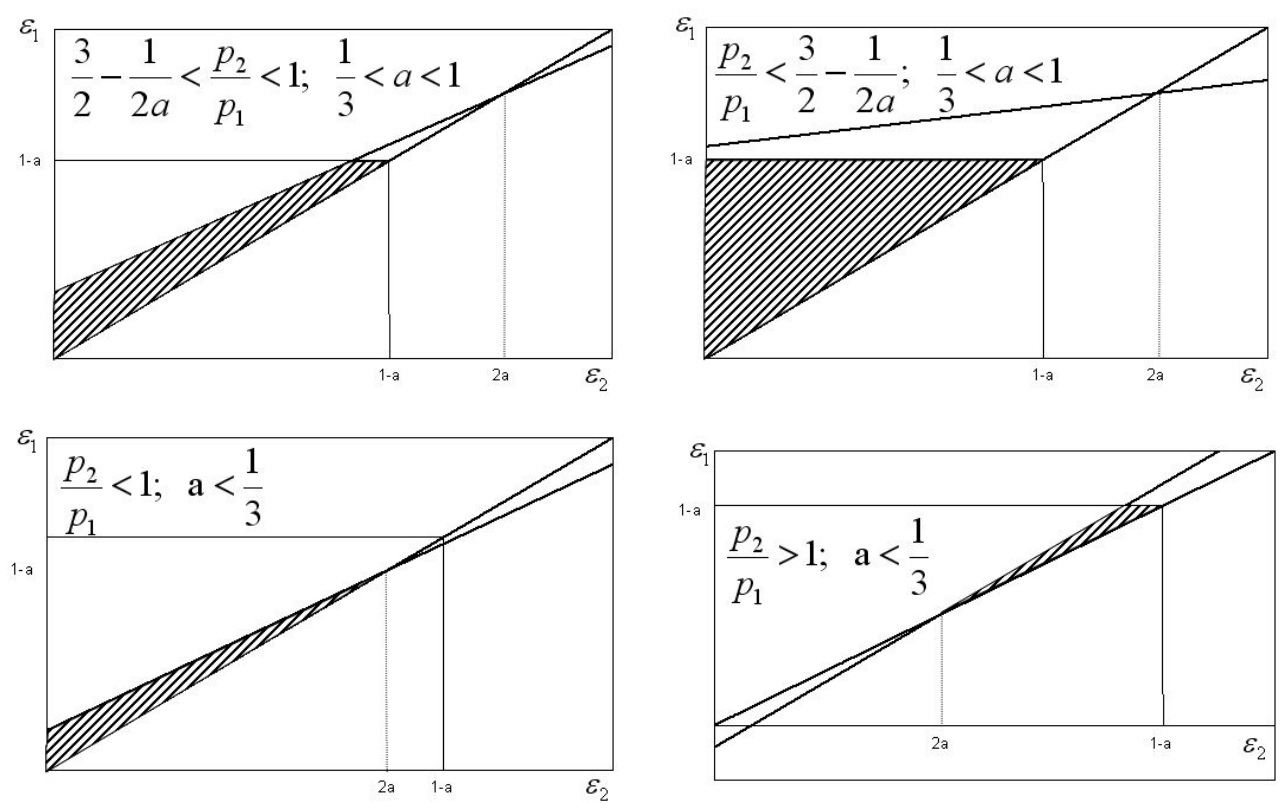

Figure 2: Sub spaces (shaded areas) in the $\left(\epsilon_{1}, \epsilon_{2}, a, p_{2} / p_{1}\right)$ space for which the inequalities of Equation (14) are fulfilled. 


$$
=\left\|\overline{\overline{\mathbf{P}}}_{\mathbf{W}}\right\|_{1} \underset{\left(p_{1}, \ldots, p_{k}\right) \rightarrow(1, \ldots, 1)}{\longrightarrow} w_{N_{1}, \ldots, N_{k}}
$$

with $\mathbf{w}$ denoting the $k$-dimensional mission criticality tensor and $\overline{\overline{\mathbf{P}}} \equiv \prod_{j=1}^{k} \tilde{P}^{(j)}$ the $k$-dimensional component reliability probability tensor. The tensor in the 1-norm has components $(\overline{\overline{\mathbf{P}}} \mathbf{w})_{i_{1}, \ldots, i_{k}}=\tilde{P}_{i_{1}}^{(1)} \cdots \tilde{P}_{i_{k}}^{(k)} w_{i_{1}, \ldots, i_{k}}$.

This mission criticality tensor in Equation (15) obeys the constraints

$$
0 \leq w_{j_{1}, \ldots, j_{k}} \leq 1, \quad w_{0, \ldots, 0}=0 \quad \text { and } \quad w_{j_{1}, \ldots, j_{l}, \ldots, j_{k}} \leq w_{j_{1}, \ldots, j_{l}+1, \ldots, j_{k}}
$$

for all $l \in\{1, \ldots, k\}$. The last of these three constraints is the monotonicity constraint defining coherent systems and ensuring, as can be proved, that the addition of any extra component increases system effectiveness.

\section{Summary and discussion}

We developed a measure of system effectiveness which is the product of component reliability probability and mission criticality summed over all system states reached by component failure. In the limit of vanishing component failure rate, this metric equals an intuitive, reliability ignorant measure of system effectiveness. We illustrated, by way of example, that because of the coupling of component reliability and mission criticality, reliability independent and reliability dependent evaluation of system performance can result in option preference reversal.

In defining the measure of reliability dependent system effectiveness we made a series of assumptions, most of which can be relaxed. Firstly, we constrained ourselves to discussing coherent systems. The measure of system effectiveness can be equally used in the assessment of incoherent systems, which are described by mission criticality tensors that violate the monotonicity constraint in Equation (16). 
Secondly, we assumed that the probabilities for component reliability do not depend on the number of components in the system or the mission profiles. In military systems these assumptions are often not fulfilled. For instance, an enemy may only consider attacking when faced by a small opposition force; thus by increasing the number of components a threshold will be reached above which attacks become less likely. On the other hand, component reliability may decrease because larger systems can have larger signatures and thus may be easier to detect (and hence easier to target). Similarly, different environments in which a system is to operate may cause different levels of degradation; that is, depending on the missions to perform, component failure rates can vary. We can account for these dependencies by replacing the constant reliability probabilities $p_{i}$ in Equation (15) with distributions that depend on both component mix and mission profile: $p_{i} \rightarrow p_{i}(\mathbf{N},[\mathbf{w}])$, thus $\overline{\overline{\mathbf{P}}} \rightarrow \overline{\overline{\mathbf{P}}}(\mathbf{N}, \mathbf{w})$. Similar to introducing the mission criticality matrix in Equation (9), we can also allow the component reliability probability tensor to be of more general structure than a dyadic product of binomial component reliability probability vectors $\tilde{P}^{(k)}$.

Thirdly, we considered the performance of systems in a single mission only. It is more realistic to assess system effectiveness against a range of missions, which may have different priorities according to a weight functional $f[\mathbf{w}] .^{1}$ As the set of possible missions may not be countable, a more general expression for system effectiveness will include a (potentially functional) integral over all mission tensors weighted by $f[\mathbf{w}]$ :

$$
\hat{E}(\mathbf{N}) \equiv \int D \mathbf{w} f[\mathbf{w}]\|\overline{\overline{\mathbf{P}}}(\mathbf{N}, \mathbf{w}) \mathbf{w}\|_{1} .
$$

The expression in Equation (17) can even be further generalised, to include, for instance, systems that do not only differ in the number and mix of components but also in some continuous variables. For instance, two courier services may differ in turnaround times or the way the courier vehicle fleet

${ }^{1}$ Selection and weighting of appropriate missions are, inter alia, research areas in the field of scenario analysis. 
is managed. For the purpose of generalisation we can replace the component reliability probability tensor $\overline{\mathbf{P}}$ with a more general probability tensor, $0 \leq \overline{\overline{\mathbf{S}}}\left[\mathbf{S} \rightarrow \mathbf{S}^{\prime}, \mathbf{w}\right] \leq 1$. Here $\overline{\overline{\mathbf{S}}}\left[\mathbf{S} \rightarrow \mathbf{S}^{\prime}, \mathbf{w}\right]$ describes the probability of a system with design state $\mathbf{S}$ to change into state $\mathbf{S}^{\prime}$. To complete this generalisation we need to replace the summations in the 1-norm of Equation (17) with integrations over all systems states $\mathbf{S}^{\prime}$ that can be reached from the design state $\mathbf{S}$. As these states can be described through variations of system parameters (such as component mix or system management variables), the summations in Equation (17) need to be replaced with integrations over (continuous) system parameters, or, more generally, with a functional integral $\int D \mathbf{S}^{\prime}$ over all possible systems states. Hence, the most general formulation of system effectiveness in our approach is

$$
\hat{E}[\mathbf{S}] \equiv \iint D \mathbf{S}^{\prime} D \mathbf{w} f[\mathbf{w}] \overline{\overline{\mathbf{S}}}\left(\mathbf{S} \rightarrow \mathbf{S}^{\prime}, \mathbf{w}\right) \mathbf{w} .
$$

Equation (18) represents a generating functional which can be used to define a range of effectiveness-based system measures of performance. These fall into two main classes:

1. flexibility measures which are derivatives of $\hat{E}[\mathbf{S}]$ with respect to mission criticality variables; and

2. robustness measures which are derivatives of $\hat{E}[\mathbf{S}]$ with respect to system variables.

\section{References}

[1] P. W. Bridgman. Dimensional Analysis. Yale University Press, 1922. $\mathrm{C} 762$

[2] P. C. Fishburn. Additive Utilities with Incomplete Product Set: Applications to Priorities and Assignments. Operations Research Society of America, Baltimore, 1967. C762 
[3] J. F. Meyer, On Evaluating the Performability of Degradable Computing Systems, IEEE Transactions on Computers, Vol. c-29, 1980, 720-731. C763

[4] D. M. Nicol, W. H. Sanders and K. S. Trivedi. Model-Based Evaluation: From Dependability to Security, IEEE Transactions on Dependable and Secure Computing, Vol. 1, No. 1, January-March 2004. C762

[5] A. Reibman, R. Smith and K. Trivedi, Markov and Markov reward model transient analysis: An overview of numerical approaches, European Journal of Operational Research, Vol. 40, 1989, 257-267. C762

[6] N. Storey, Safety-Critical Computer Systems, Addison Wesley Longman Inc., 1996. C762

[7] K. Yoon and C. L Hwang, Multi Attribute Decision Making: An Introduction, Sage, 1995. C762 